\title{
Star Formation in the Local Universe from the CALIFA sample: calibration and contribution of disks to the SFR density
}

\author{
Cristina Catalán-Torrecilla ${ }^{1}$, Armando Gil de Paz ${ }^{1}$, \\ África Castillo-Morales ${ }^{1}$, Jorge Iglesias-Páramo ${ }^{2}$, \\ Sebastián F. Sánchez ${ }^{2,3}$ and CALIFA Collaboration \\ ${ }^{1}$ Departamento de Astrofísica y CC. de la Atmósfera, Universidad Complutense de Madrid, \\ E-28040, Madrid, Spain, email: ccatalan@ucm.es \\ ${ }^{2}$ Instituto de Astrofísica de Andalucía-CSIC, Glorieta de la Astronomía, 18008 Granada, Spain \\ ${ }^{3}$ Instituto de Astronomía,Universidad Nacional Autonóma de México, 04510, México
}

\begin{abstract}
The study of the star formation rate (SFR) is crucial for understanding the birth and evolution of the galaxies (Kennicutt 1998), with this aim in mind, we make use of a wellcharacterized sample of 380 nearby galaxies from the CALIFA survey that fill the entire colormagnitude diagram in the Local Universe. The availability of wide-field CALIFA IFS ensures a proper determination of the underlying stellar continuum and, consequently, of the extictioncorrected $\mathrm{H} \alpha$ luminosity. We compare our integrated $\mathrm{H} \alpha$-based SFRs with single and hybrids tracers at other wavelengths found in the literature (Calzetti 2013). Then, we provide a new set of single-band and hybrid calibrators anchored to the extinction-corrected $\mathrm{H} \alpha$ luminosities. In the case of the hybrid calibrators we determine the best fitting $a_{I R}$ coefficients for different combinations of observed (UV or $\mathrm{H} \alpha$ ) and dust-reprocessed ( $22 \mu \mathrm{m}$ or TIR) SFR contributions (where SFR $\propto \mathrm{L}_{o b s}+a_{I R} \times \mathrm{L}[\mathrm{IR}]$ ). This analysis allow us to provide, for the first time, a set of hybrid calibrations for different morphological types and masses. These are particularly useful in case that the sample to be analyzed shows a different bias in terms of morphology or, more commonly, luminosity or stellar mass. We also study the dependence of this coefficient with color and ionized-gas attenuation. The distributions of $a_{I R}$ values are quite wide in all cases. We found that not single physical property can by itself explain the variation found in $a_{I R}$.

Finally, we explore the spatial distribution of the SFR by measuring the contribution of disks to the total SFR in the Local Universe. Our preliminary spatially-resolved analysis shows that the disk to total (disk + spheroidal component) SFR ratio is on average $\sim 88 \%$. The use of the $2 \mathrm{D}$ spectroscopic data is critical to properly determine the $\mathrm{H} \alpha$ luminosity function and SFR density in the Local Universe per galaxy components, the ultimate goal of this project.
\end{abstract}

Keywords. galaxies: spiral - galaxies: evolution - galaxies: star formation

\section{Acknowledgements}

This study makes uses of the data provided by the CALIFA survey. CALIFA would like to thank the IAA-CSIC and MPIA-MPG. C. C.-T. thanks the support of the Spanish Ministerio de Educación, Cultura y Deporte by means of the FPU fellowship program.

\section{References}

Kennicutt, R. C. 1998, ARA\&A, 36, 189

Calzetti, D. 2013, Star Formation Rate Indicators, ed. J. Falcón-Barroso \& J. H. Knapen, 419 